Cahiers de recherches médiévales

\title{
Au miroir du Tuchinat
}

Relations sociales et réseaux de solidarité dans les communautés languedociennes à la fin du $\mathrm{XIV}^{\mathrm{e}}$ siècle

\section{Vincent Challet}

\section{(2) OpenEdition \\ 12 Journals}

Édition électronique

URL : https://journals.openedition.org/crm/1563

DOI : $10.4000 / \mathrm{crm} .1563$

ISSN : 1955-2424

Éditeur

Honoré Champion

\section{Édition imprimée}

Date de publication : 15 mars 2003

Pagination : 71-87

ISSN : $1272-9752$

\section{Référence électronique}

Vincent Challet, «Au miroir du Tuchinat », Cahiers de recherches médiévales [En ligne], 10 | 2003, mis en ligne le 08 octobre 2007, consulté le 15 décembre 2022. URL : http://journals.openedition.org/crm/ 1563 ; DOI : https://doi.org/10.4000/crm.1563

Ce document a été généré automatiquement le 15 décembre 2022.

Tous droits réservés 


\title{
Au miroir du Tuchinat \\ Relations sociales et réseaux de solidarité dans les communautés languedociennes à la fin du XIV ${ }^{\mathrm{e}}$ siècle
}

\author{
Vincent Challet
}

1 Longtemps, l'insurrection des Tuchins qui agita l'Auvergne puis les trois sénéchaussées du Languedoc entre 1381 et 1384 ne fut considérée par les historiens que comme une révolte de marginaux poussés par la misère, un regroupement de «bandes d'asociaux affamés " ${ }^{1}$ sans appui, sans soutien et sans programme. Cette vision fut largement et durablement influencée par la seule chronique nous ayant conservé le souvenir du Tuchinat, celle de Michel Pintoin, qui applique aux rebelles languedociens le terme d'abjectissimi et compare l'apparition des Tuchins à celle d'une "nuée de vers" ${ }^{2}$. Il convient toutefois de noter que jamais le Religieux de Saint-Denis n'utilise pour désigner ces révoltés un mot dont le sens puisse être approché de celui de marginal mais se contente de leur attribuer un qualificatif qui les stigmatise d'un point de vue moral et religieux, sans véritable contenu social. D'ailleurs, la longue durée du mouvement, son implantation et son extension géographique peu commune dans le cadre de révoltes médiévales, ce que Claude Gauvard appelle « la pugnacité des Tuchins en guérilla ", conduisent à s'interroger de façon plus précise sur la prétendue marginalité de ces Languedociens. Encore faudrait-il pouvoir s'entendre sur le sens que l'on donne à cette notion de marginalité : il n'est en effet pas tout à fait sûr qu'il faille qualifier de marginal tout individu « dont le mode de vie se présente comme anormal, c'est-à-dire ne respecte pas les normes en vigueur dans la société »". Il semble en effet, en particulier dans le cadre des communautés paysannes, que le marginal corresponde davantage à un individu isolé, privé de ces "amis charnels» dont l'appui semble fondamental dans les sociétés médiévales, coupé des réseaux de sociabilité sans lesquels un être ne peut que très difficilement survivre. Or, les Tuchins peuvent bien être définis comme des anormaux dans la mesure où ils s'insurgent bien contre une norme, qu'elle ait été établie par les pouvoirs urbains ou par le lieutenant du roi en Languedoc: ceci ne suffit pourtant pas à en faire des marginaux ${ }^{5}$. En outre, l'assimilation du révolté au marginal - de même que le lien entre marginalité et 
criminalité ${ }^{6}$ parait davantage découler d'une vision des chroniqueurs du Moyen Âge que de la réalité des sources.

2 Ce vocable de Tuchins ne contribua pas peu à renforcer l'idée que cette rébellion méridionale s'inscrivait, non pas au cœur même de la société, mais dans des marges qui étaient tout autant géographiques que sociales. Si l'on s'en tient en effet à une stricte étymologie, le Tuchin est celui qui tient la "touche»", ce qui a pu amener Robert Fossier à qualifier les Tuchins de "fugitifs qui tiennent le maquis » et a contribué à enraciner cette image de maquisards occitans. Toutefois, il convient d'observer que ce terme n'a pas une origine méridionale et que sa première occurrence se trouve dans une lettre de rémission accordée à deux habitants d'une paroisse du Cotentin pour le meurtre de Jean Moissant « qui estoit devenu brigant ou tuchin de bois ». Cet homicide, commis vers 1356-1357, doit être replacé dans le contexte des actes d'autodéfense perpétrés par les communautés paysannes du Cotentin contre les exactions des garnisons anglo-navarraises. La première occurrence du terme fait donc apparaître une triple équivalence : en Normandie et dès le milieu du XIV ${ }^{e}$ siècle, le Tuchin n'est pas seulement celui qui se réfugie dans la « touche", mais aussi celui qui prend les armes à l'instar du brigand et enfin celui qui tente de s'opposer aux razzias des compagnies selon des modalités qui semblent proches des «partisans» du XV $\mathrm{XV}^{\mathrm{e}}$ siècle ${ }^{10}$. Ce n'est toutefois qu'un peu plus tard, dans l'Auvergne des années 1360, que naît véritablement le Tuchinat si l'on veut bien entendre par là non plus la juxtaposition d'initiatives individuelles mais un mouvement plus ou moins structuré, parvenant à se doter d'un minimum d'organisation et qui se donne comme objectif premier de lutter contre les compagnies anglo-gasconnes. Le premier fait avéré de Tuchinat en Auvergne suit d'ailleurs immédiatement la prise de Brioude par les routiers de Seguin de Badefol et atteste de la présence d'une bande de Tuchins à Vieille-Brioude ${ }^{11}$. À la différence des meurtres improvisés de gens de compagnie commis par des paysans retirés dans les bois ou se défendant contre des pillards et qu'il est possible de retrouver dans toutes les régions du royaume de France en butte aux exactions des routiers, "l'action des Tuchins est permanente et organisée ${ }^{12}$ et l'autodéfense prend ici la forme d'une societas dont l'élément fondamental demeure le serment prêté à un capitaine.

Cette existence d'un serment a considérablement frappé les contemporains, à commencer par le Religieux de Saint-Denis pour lequel les Tuchins «s'étaient réunis et engagés par des serments terribles à ne plus courber la tête sous le poids des subsides ${ }^{13}$, et les clercs de la chancellerie royale ne manquent pas, dans les lettres de rémission accordées pour fait de Tuchinat, de souligner cet aspect en usant de l'expression secta Tuchinorum ou en stigmatisant un coupable en affirmant qu'il est de sacramento Tuchinorum ${ }^{14}$. Le serment ne constitue cependant pas une originalité de ce mouvement mais au contraire une forme si élémentaire de solidarité villageoise qu'il semble attesté, à en croire Wace, lors de la révolte des paysans normands de $996^{15}$. En ce qui concerne le Tuchinat languedocien, la teneur même de ce serment, d'une simplicité extrême, nous est connue à travers le témoignage d'un laboureur de Bagnols-sur-Cèze, Jacques Fabre, qui déclare avoir été contraint sous la menace de jurer d'être bon et fidèle aux Tuchins ${ }^{16}$. Cette formule semble largement empruntée à celle du serment que devait prêter le capitaine de la ville lors de son entrée en fonction ${ }^{17}$ et se retrouve notamment en usage dans les compagnies de partisans normands dans les années 
$1420^{18}$. La seule mention de ce serment témoigne, à l'évidence, de l'hiatus existant entre la vision des élites imposant un qualificatif péjoratif puisqu'assimilable à celui de «brigand » et la réalité d'un mouvement qui participe des solidarités paysannes. Si la façon de désigner des révoltés constitue une arme dans l'arsenal politique mobilisé pour les combattre, c'est bien au pouvoir royal que revint le dernier mot. Non seulement, le terme de Tuchin s'imposa pour désigner ceux qui participèrent à la rébellion languedocienne des années 1381-1384 mais il devint si rapidement une injure que Charles VI dut faire proclamer à Nîmes, après la fin de la révolte, « que nul ne feüst si hardi d'appeler aucune personne touchin $»^{19}$.

4 À l'inverse de cette impression de marginalité, les témoignages recueillis en 1385 à l'occasion d'un procès engagé contre les procureurs de Bagnols-sur-Cèze ${ }^{20}$ révèlent que le Tuchinat s'appuie sur les liens de sociabilité qui sous-tendent la vie communautaire des villages de la sénéchaussée de Beaucaire. Ils permettent d'ailleurs d'établir que, conformément à ce que l'on pouvait soupçonner, les révoltés ne se désignent jamais sous le terme de Tuchins ${ }^{21}$ mais sous celui de companhos qui renvoie au contraire à une intégration du mouvement au sein des communautés villageoises. Ce terme de compahnos est celui qu'utilisent les rédacteurs des comptes consulaires de Saint-Flour dès $1380^{22}$, de Najac en $1383^{23}$ ou encore de Conques entre 1386 et $1391^{24}$, tous documents rédigés en langue vernaculaire. Dans les actes judiciaires adoptant le latin, c'est le terme de socius qui apparaît alors comme à l'occasion du témoignage de Jean Paumier, prêtre de Bagnols-sur-Cèze, qui affirme que dans le village de Saint-Gervais ${ }^{25}$ se trouvaient plures Tuchini qui tunc socii nominabantur ${ }^{26}$. Non seulement les révoltés se voient comme des compagnons - sous-entendant ainsi la permanence d'un lien social et l'existence d'un devoir d'entraide - mais ils sont perçus de la même façon par ceux qui, au sein des communautés, les côtoient quotidiennement.

5 Le Tuchinat est avant tout la manifestation d'une solidarité villageoise élémentaire à l'encontre des menaces que font peser depuis les années 1360 les courses des routiers et autres gens d'armes, de quelque bord qu'ils puissent être. Aux yeux des paysans, la différence entre les compagnies anglo-gasconnes et les armées royales était d'autant moins nette qu'elles adoptaient le même comportement vis-à-vis de la population civile $^{27}$ et que l'habitude se prit, en Languedoc plus qu'ailleurs semble-t-il, de retenir au service du roi de France des capitaines de compagnie ${ }^{28}$. Ce que le pouvoir qualifia ultérieurement de révolte ne procède donc au départ que d'un réflexe de survie visant à soustraire les biens essentiels de la communauté - hommes, réserves de blé, chevaux et cheptel - à la convoitise des pillards. Honoré Bovet devait, quelques années plus tard, porter sur ces campagnes militaires un jugement que partageaient sans doute bien des Languedociens :

«A Dieu ne plaise de mettre es cueurs des rois de ordrener comment en toutes guerres, les poures laboureux soient seurs, car au jour duy, toutes les guerres sont contre les poures gens, contre les buefs et contre les vaches, et ce nest mie guerre mes me semble pillarderie. $»^{29}$

Le Tuchinat calqua son organisation sur les structures qui permettaient à chaque communauté d'assurer la défense de son territoire et, de ce fait, il se présente comme la poursuite des diverses stratégies de lutte élaborées par les villageois au cours de la Guerre de Cent $\mathrm{Ans}^{30}$. En effet, vers 1380, les Languedociens comprirent que le combat contre les compagnies ne pouvait être mené efficacement que s'il dépassait le cadre 
strictement défensif de l'appel aux voisins en cas d'attaque soudaine. Cette attitude qui s'apparentait au vieux «cri de commune » et incitait les habitants à porter secours à l'un des leurs en cas d'agression ${ }^{31}$ se retrouve dans les trois sénéchaussées de Languedoc à partir de 1360. Pour ne prendre ici qu'un seul exemple, lorsqu'en 1361, des membres de la grande Compagnie lancèrent un raid sur le petit village de Chusclan, ils réussirent dans un premier temps à s'emparer d'une grande quantité de bétail. Cependant, les villageois s'armèrent, tuèrent l'un des routiers et parvinrent à récupérer la majeure partie de leur bien ${ }^{32}$. Mais un tel comportement ne pouvait être qu'une réponse ponctuelle et parfois dérisoire à la menace permanente que faisaient peser sur les communautés villageoises les raids perpétrés par les compagnies. Une prise de conscience de la nécessité d'une défense commune qui reposerait sur des regroupements de communautés s'opéra progressivement chez les habitants de la province. Elle explique le changement d'échelle qui se mit en place à partir de septembre 1381 et de l'arrivée des troupes du nouveau lieutenant du roi en Languedoc, Jean de Berry. L'opposition qui se manifeste alors n'est pas que de nature politique ou sociale; elle est aussi mouvement d'autodéfense face aux ravages opérés par les troupes à la solde du duc de Berry ${ }^{33}$. Defendere patriam contra inimicos et latrunculos, voilà ce que demandaient les États du Languedoc au lieutenant du roi depuis bientôt vingt ans; voilà l'objectif premier que reprennent les Tuchins en 1381.

Dans la sénéchaussée de Beaucaire, le groupe de Tuchins le plus actif qu'il soit possible de repérer se constitua autour de Bagnols-sur-Cèze. Il regroupa assez vite quatre compagnies d'une trentaine de membres au maximum et disposant d'une certaine autonomie de commandement et d'action. Chaque ensemble était placé sous la responsabilité d'un capitaine qui recueillait les serments des nouveaux venus, planifiait les opérations de son groupe, veillait à la discipline interne et procédait à la distribution du butin. La répartition des Tuchins entre ces quatre compagnies laisse deviner un recrutement obéissant à une logique géographique et s'appuyant sur des liens de voisinage antérieurs au mouvement. En effet, tandis que les hommes originaires des localités situées à l'est de la viguerie de Bagnols-sur-Cèze se rangent sous la bannière de Verchière, lui-même issu de Vénéjan ${ }^{34}$, les compagnons provenant des villages de l'ouest de cette viguerie s'intègrent dans les rangs du groupe commandé par Bernard Régis qui réside à Saint-Michel-d'Euzet ${ }^{35}$. En outre, l'ensemble de ces villages se trouve dans un rayon d'une trentaine de kilomètres autour de Bagnols-surCèze. Sur les cinquante-huit Tuchins dont nous possédons à la fois le nom ou le surnom et le lieu d'origine, cette règle ne souffre que d'une seule exception. Elle concerne les révoltés originaires de la ville même de Bagnols-sur-Cèze qui se répartissent entre ces deux compagnies sans que, faute d'informations complémentaires, il soit possible de déterminer les causes de ces différents rattachements. Enfin, les deux autres compagnies, dirigées par Vachon et Ferragut ${ }^{36}$, privilégient un recrutement plus méridional puisque leurs hommes sont originaires de la région d'Uzès et qu'eux-mêmes sont en relation avec les consuls de cette ville.

8 Les modalités selon lesquelles s'exerce le partage du butin révèlent également la solidarité et l'égalité des compagnons entre eux puisque chaque individu ayant participé à une opération donnée reçoit une quantité égale, à l'exception des capitaines qui se voient attribuer une part double. Ce procédé est notamment utilisé en ce qui concerne la répartition du blé trouvé dans le château d'Aiguèze, lorsqu'en septembre 
1382 cette puissante forteresse qui domine les bords de l'Ardèche tombe entre les mains des Tuchins. Après un recensement préalable des combattants et des quantités de céréales saisies, les capitaines désignèrent un Tuchin qui savait lire et écrire pour faire la distribution et inscrire sur une liste au fur et à mesure les noms des hommes qui avaient déjà touché leur part. Chaque compagnon qui avait participé à l'assaut d'Aiguèze eut droit à trois salmées ${ }^{37}$ de blé tandis que les quatre capitaines et le bayle de Pons Biordon qui avait trahi son seigneur et livré le château aux révoltés se virent attribuer six salmées de ce même blé. Ce partage à la fois rigoureux et équitable révèle un véritable sens de l'organisation, une évidente égalité entre les compagnons ainsi qu'une absence de hiérarchie, hormis celle qui place les capitaines au-dessus de leurs hommes.

9 L'utilisation de ce blé par les compagnons démontre qu'ils continuèrent, au cours même du mouvement, à maintenir les liens qui les unissaient à leurs communautés. Pour nombre d'individus, la participation à la révolte reste en effet occasionnelle et entrecoupée de longues périodes de travail, qu'il s'agisse de curer les fossés de la ville de Bagnols-sur-Cèze pour le compte des procureurs de la ville ou de se louer à des propriétaires terriens à l'occasion des moissons ou des vendanges. La translation par les Tuchins dans leur village d'origine de leur part du blé saisi à Aiguèze reste l'indice d'une forte intégration au sein de la société villageoise bien plus que d'une rupture visà-vis de celle-ci. De telles opérations sont attestées individuellement pour de nombreux compagnons, à l'exemple de Jean Portal réquisitionnant une barque pour traverser l'Ardèche et entreposer neuf ou dix setiers de blé à son domicile situé dans le Vivarais ${ }^{38}$. En outre, les capitaines procédèrent également à des transferts collectifs en regroupant les parts de plusieurs Tuchins issus de la même localité: un convoi organisé par Ferragut permit ainsi d'acheminer environ cinquante salmées - soit la rétribution d'une quinzaine d'hommes - en direction d'Uzès tandis que Bernard Régis expédia la même quantité de blé à Saint-Michel-d'Euzet, où lui et plusieurs hommes de sa compagnie résidaient. Les céréales ainsi recueillies ne furent pas systématiquement dissipées mais purent servir au règlement de rentes ou de cens restés impayés en raison de la difficile conjoncture économique : Jean Maistre, forgeron de Saint-Micheld'Euzet, remit quatre salmées de blé et cinq de seigle à un propriétaire de Bagnols-surCèze pour le loyer d'une terre qu'il exploitait. De même, Mondon Petit, tuchin qui faisait partie de la compagnie de Bernard Régis, proposa à un drapier de Bagnols-surCèze de régler en blé la tunique qu'il faisait réaliser pour sa femme, ce que le drapier refusa pour ne pas être accusé de recel.

Le Tuchinat révèle également son insertion au sein des communautés villageoises par la récupération et la réinterprétation de rites fondateurs d'une sociabilité qui trouve, pour la première fois, à s'exprimer au cœur de l'espace urbain. La révolte est ainsi une réappropriation symbolique de la ville et de ses lieux de pouvoir, comme l'atteste la célébration du mariage de Bernard Régis dans l'église paroissiale de Bagnols-sur-Cèze en un moment où les rebelles sont les véritables maîtres de cette localité. Le déroulement de ces noces à l'intérieur même de la ville et non pas à Saint-Micheld'Euzet, village d'origine de l'époux, est déjà une manifestation de cette emprise des Tuchins sur la cité. En outre, au cours du banquet nuptial qui s'ensuivit, les invités dont bon nombre étaient les compagnons de lutte de Bernard Régis - consommèrent $\mathrm{du}$ vin et $\mathrm{du}$ pain en provenance des demeures nobiliaires de Bagnols-sur-Cèze. Le Tuchinat inscrit ainsi l'un des rituels fondateurs de la solidarité villageoise dans un 
espace qui lui est foncièrement hostile tout en faisant reposer le poids de cette cérémonie sur ses principaux adversaires contraints, en l'occasion, de financer sur leurs propres deniers la refondation du lien social.

11 Il s'agit bien là d'un détournement par les révoltés d'un rituel pacifique et consensuel réutilisé en l'occurrence à des fins politiques. Cet aspect du Tuchinat est également mis en lumière par l'entrée des compagnons dans Bagnols-sur-Cèze, parfaitement orchestrée par leurs capitaines de manière à réoccuper l'espace urbain et à faire de la ville une véritable scène de théâtre qui sert de caisse de résonance aux revendications des Tuchins. Bernard Régis, Vachon, Verchière et Ferragut choisirent pour leur démonstration de force un jour où Guillaume de Beaufort, comte d'Alès, vicomte de Turenne et seigneur de Bagnols-sur-Cèze, se trouvait précisément dans la ville. Ils firent alors défiler leurs hommes au son du tambour et des trompettes, bannières déployées ${ }^{39}$, et occupèrent la grande rue qui conduisait jusqu'à l'église paroissiale et à la principale place de la citée ${ }^{\prime 0}$. C'est donc à une véritable prise de possession de la ville que se livrent les révoltés à l'occasion de leur entrée. L'ordre dans lequel ils se présentent, les bannières déployées dans la rue qui permettent pour les témoins une claire identification des compagnies et de leur chef et jouent le « rôle de légitimation de l'action du groupe " ${ }^{41}$, l'environnement sonore qui imprègne cette cérémonie ${ }^{42}$, tout concourt à faire de cette entrée un véritable défilé militaire et réfute la vision de bandes anarchiques composées de gueux. L'organisation qui prélude à cette invasion de l'espace urbain et son déroulement même en font un rituel villageois qui emprunte sans doute au modèle de la réception des gens d'armes au cours des montres et rappelle, sur un mode mineur, celle des papes ou des rois dans les villes languedociennes ${ }^{43}$. Les Tuchins s'imposent ainsi aux autorités urbaines, constituées en l'occurrence par les quatre procureurs de la communautée, et la force du rituel se manifeste d'autant plus que l'entrée des Tuchins contraint le vicomte de Turenne à se rendre dans la grande rue de Bagnols-sur-Cèze pour y inspecter les bannières des révoltés, alors même que son hôtel particulier ne se trouve pas dans cette artère ${ }^{45}$. La démonstration de force des Tuchins conduit donc Guillaume de Beaufort à venir à eux et non pas l'inverse.

12 L'action du vicomte de Turenne ne se limite d'ailleurs pas à s'avancer à la rencontre des révoltés. Il n'hésite pas en effet à entamer le dialogue avec Verchière, l'un des anciens officiers de son père devenu capitaine tuchin : à ce rebelle qui lui assure que l'action de ses hommes n'est pas dirigée contre lui - et le Tuchinat ne semble effectivement pas un mouvement anti-seigneurial -, il répond qu'il n'en a pas douté un instant et va même jusqu'à dire publiquement que les compagnons agissaient bien ${ }^{46}$. Pour avoir prononcé des paroles quasi identiques, un brassier de Beaucaire fut, quelque temps plus tard, condamné à une amende de dix francs d'or ${ }^{47}$. La création de cet espace de familiarité à l'initiative du vicomte de Turenne surprend d'autant plus que le seigneur de Bagnolssur-Cèze, pourtant nommé par le duc de Berry capitaine général de la sénéchaussée de Beaucaire et chargé de lutter contre les Tuchins, la prolonge par une invitation lancée aux quatre capitaines présents dans la ville à partager avec lui un verre de vin dans sa propre demeure. Il accomplissait ainsi vis-à-vis des révoltés l'un des gestes élémentaires de sociabilité et, en buvant avec les compagnons, les reconnaissait publiquement comme des commensaux, ou à tout le moins, comme des membres à part entière d'une communauté regroupant l'ensemble des membres de sa seigneurie. Le 
geste de Guillaume de Beaufort est d'autant plus étonnant qu'il s'agit d'un rite d'agrégation qui transgresse les barrières sociales et semble très inhabituel dans une société où toute tentative d'abolition de la hiérarchie est vue comme une mise en danger d'un ordre tout à la fois politique et divin. Le bourreau Capeluche paya justement de sa vie le fait d'avoir cru pouvoir créer cet espace de familiarité avec le duc de Bourgogne Jean sans Peur en lui offrant un gobelet de vin, témoignant ainsi de sa volonté de boire avec un membre de la famille royale ${ }^{49}$. Que certains contemporains aient pu lier la chute de Capeluche à la transgression de l'un des tabous les plus absolus de la société médiévale, le meurtre de la femme enceinte ${ }^{50}$, ne change rien: qu'il ait voulu s'élever au-dessus de sa condition sociale ou qu'il ait éventré une femme enceinte, "Capeluche s'est exclu et est exclu de la communauté ". A contrario, le partage du vin entre le vicomte de Turenne et les capitaines révoltés souligne la profonde intégration de ces derniers au sein de leurs communautés respectives. Cette marque de sociabilité se révèle toutefois factice puisque Guillaume de Beaufort, avant de quitter la ville, reproche vertement aux boni homines de Bagnols-sur-Cèze d'avoir permis l'entrée de ceux qu'il qualifie de "ribauds» et menace de faire pendre les bourgeois et les procureurs de la cité pour complicité avec les révoltés.

Ce comportement qui vise à nier en privé la force de cet acte public semble avoir été perçu par Ferragut qui, requis par le vicomte de Turenne de venir boire un verre de vin avec les autres capitaines tuchins, déclina ostensiblement cette invitation ${ }^{52}$. Ce refus est très certainement un signe de défiance vis-à-vis de celui qui représente l'autorité du duc de Berry dans la région mais il est sans doute aussi l'indice du refus de la transgression de règles sociales non-écrites qui interdisent la célébration de rites de convivialité entre inégaux. "Prendre un pot", cet acte fondateur de la sociabilité villageoise, ne peut précisément se faire qu'entre soi, entre frères ou compagnons qui partagent les mêmes valeurs. Frère Guillaume Vital, du couvent des Frères Mineurs de Bagnols-sur-Cèze, ne dit pas autre chose lorsqu'il relate que, croisant par hasard au détour d'un chemin, son ancien confrère Jean de Ferran, franciscain défroqué qui avait rejoint les Tuchins, ce dernier le salua et l'invita boire à un verre, car il le connaissait ${ }^{53}$. C'est bien la connaissance de l'autre et sa fréquentation antérieure qui rendent possible ce partage de la boisson qui ne saurait suffire en lui-même à créer une relation d'amitié. Un autre épisode signale les enjeux fondamentaux qui se nouent autour de l'offre et de l'acceptation ou non du verre de vin. Un jour que Verchière et Ferragut, escortés par plusieurs boni homines de Bagnols-sur-Cèze se rendaient dans cette ville pour une conférence de paix avec les envoyés du duc de Berry, ils firent une halte dans le village de Saint-Paulet-de-Caisson ${ }^{54}$ où ils furent accueillis par Pierre Gaydell. Ce noble, officier du vicomte de Turenne et ancien capitaine de Bagnols-sur-Cèze pour le compte de ce dernier, offrit aux chefs tuchins un verre de son vin blanc qu'ils acceptèrent. Mais Verchière, après l'avoir goûté, refusa d'en boire davantage, déclarant publiquement qu'il n'était pas bon ${ }^{55}$. Cet acte n'est en rien anodin et le prêtre d'Aiguèze qui assista à la scène ne manque pas de le rapporter aux commissaires royaux chargés de recueillir son témoignage, preuve qu'il y a décelé une véritable provocation de la part de Verchière. Offrir à boire constitue toujours un geste de bienvenue et, a contrario, refuser le verre de vin qui vous est tendu est une grave offense qui, en temps ordinaire, met en danger la vie de celui qui accomplit un tel geste ${ }^{56}$. Ainsi que l'attestent de nombreuses lettres de rémission, celui qui repousse le verre de vin qui lui est tendu 
s'exclut de facto d'une communauté en devenir en négligeant d'adhérer à l'un de ses rites fondateurs. Il devient en conséquence un individu isolé que ne protègent plus les règles élémentaires de la solidarité villageoise et expose son corps à la violence des autres. Or, dans le cas qui nous préoccupe, le rituel qui se met en place est exactement inverse puisque, en rejetant l'offre qui lui est faite, Verchière accomplit un geste qui le rapproche de ses compagnons et le démarque de toute complicité avec un officier du vicomte de Turenne : le rituel n'est plus ici d'exclusion mais bien d'intégration. Ce refus de boire peut donc se lire comme une inversion des modalités des relations qui unissent le seigneur et ses dépendants.

14 Les actions menées par les Tuchins révèlent également le souci des révoltés de protéger autant que faire se peut ce qui parait indispensable à la sauvegarde des communautés villageoises. La plupart des opérations dont nous connaissons les détails concernent en effet la récupération du bétail razzié à l'occasion de raids menés sur les villages par les gens d'armes de Gantonnet d'Apzac, lieutenant de Guillaume de Beaufort et chargé par ce dernier de lutter contre les Tuchins. C'est dans le courant du mois de novembre 1382 que Gantonnet d'Apzac réoccupa militairement Pont-Saint-Esprit et se présenta devant Bagnols-sur-Cèze à la tête d'une quarantaine d'hommes d'armes pour y être reçu comme capitaine au nom du vicomte de Turenne. Toutefois, avant de parvenir aux barrières de la ville, il avait pris soin de faire ravager les environs et de s'emparer du lieu de Saint-Gervais ${ }^{57}$ ainsi que de son église, dans laquelle s'étaient réfugiés les habitants. À cette occasion, les soldats de sa compagnie incendièrent plusieurs maisons du village, arrachèrent les oreilles d'un forgeron sur lequel pesaient des présomptions de recel et tuèrent deux habitants de Saint-Gervais ${ }^{58}$. Ces opérations qui visaient à terroriser la population et à couper les Tuchins des communautés villageoises eurent au contraire pour conséquence de favoriser la mise en place d'une alliance tactique entre les «menus » et les élites urbaines. Cette coalition se manifesta dans un premier temps par le refus des procureurs de Bagnols-sur-Cèze de laisser pénétrer Gantonnet d'Apzac à l'intérieur de l'espace urbain. Le lieutenant du vicomte de Turenne leur envoya alors des lettres de défi ad ignem et sanguinem ${ }^{59}$ et révéla son intention de ravager le territoire de Bagnols de telle manière que ses habitants en seraient réduits à manger des rats ${ }^{60}$. Cette situation contraignit alors les Bagnolais à s'appuyer sur les Tuchins pour assurer leur propre sécurité. C'est notamment l'argument que firent valoir les "populaires » de la ville lors des diverses assemblées générales convoquées par les procureurs afin de trouver une solution à la crise. Après l'écrasement du mouvement des Tuchins, les boni homines tentèrent d'ailleurs de dissimuler la sympathie éprouvée par les «menus » de la ville pour l'action des Tuchins en affirmant que c'était bien plus par crainte que par amour ${ }^{61}$ que Verchière, Ferragut et leurs compagnons avaient été autorisés à pénétrer dans la ville. Selon eux, une telle décision n'aurait été prise que sous la pression des laboureurs et autres ouvriers agricoles qui souhaitaient ne pas être sous la menace des Tuchins lorsqu'ils se rendaient à l'extérieur de la ville pour cultiver leurs terres.

Cette crainte d'avoir à se garder à la fois des Tuchins et des gens d'armes revient dans le témoignage de Jean Portal le vieux, bourgeois de Bagnols-sur-Cèze, qui affirme en outre que les pauperes désiraient parvenir à un accord avec les Tuchins parce que ceuxci les défendraient contre les troupes de Gantonnet d'Apzac ${ }^{62}$. L'accueil des Tuchins serait donc une réponse à la recrudescence des ravages des gens d'armes sur le territoire de Bagnols et les «menus» firent valoir que, puisque les hommes d'armes 
étaient reçus à Pont-Saint-Esprit, il était légitime de s'appuyer sur les compagnons pour contrecarrer leurs opérations. Les Bagnolais, confrontés à la menace que faisait peser sur eux l'intensification des opérations militaires, ne se contentèrent pas d'ouvrir leur cité aux révoltés et de leur offrir à la fois un asile et des possibilités de ravitaillement. La politique qu'ils mirent en place conduisit à une véritable coalition qui se donna pour objectif de protéger les biens et les personnes de la communauté bagnolaise tout en assurant dans les campagnes environnantes le minimum de sécurité nécessaire à la poursuite des travaux des champs. En effet, à Bagnols-sur-Cèze comme ailleurs, les Tuchins représentent un appoint militaire dont ne sauraient se passer les autorités urbaines. Un tel pacte entre consuls et Tuchins est ainsi attesté à Narbonne où les compagnons furent engagés comme mercenaires et stipendiés aux frais du consulat lors de la guerre que la ville eut à mener à partir de septembre 1381 contre son propre vicomte, Amaury $\mathrm{VII}^{63}$.

16 En ce qui concerne Bagnols-sur-Cèze, en une occasion au moins, Tuchins et habitants de la ville combattirent côte à côte afin de protéger le territoire de la communauté contre les entreprises de Gantonnet d'Apzac. Cet épisode se produisit au mois de février ou de mars 1383 après la conclusion d'un accord entre les Tuchins et le conseil du duc de Berry dirigé par Simon de Cramaud, évêque d'Agen ${ }^{64}$, accord au terme duquel les compagnons se voyaient accorder le droit de résider dans la ville de leur choix à condition de déposer les armes. Il s'agissait d'une première étape dans le processus initié avec l'appui des communautés par l'évêque d'Agen pour favoriser la réintégration des Tuchins au sein de l'espace social de la sénéchaussée de Beaucaire. Toutefois, cette trêve ne permit pas de mettre un terme définitif aux ravages perpétrés par les hommes d'armes de Gantonnet d'Apzac. Le lieutenant du vicomte de Turenne dévasta ainsi le territoire de Masmolène et de Tresques, deux villages situés entre Bagnols-sur-Cèze et Uzès $^{65}$. Ses troupes razzièrent les troupeaux de ces localités, emmenèrent en captivité ceux qui les gardaient et, passant par Bagnols-sur-Cèze pour regagner leurs quartiers, elles firent prisonniers plusieurs des habitants de la ville. Les gens d'armes s'emparèrent également de bœufs appartenant à Jean Coq et à Étienne Adhémar, deux marchands de Bagnols, preuve que les boni homines ne se trouvaient pas à l'abri de ces déprédations. La réaction ne se fit pas attendre et prit la forme d'une ligue entre les habitants de la ville, ceux des Tuchins qui avaient accepté la trêve et résidaient à Bagnols-sur-Cèze et enfin les Tuchins qui demeuraient sous les armes et se trouvaient au couvent des Frères Mineurs ${ }^{66}$. Le combat livré contre les gens d'armes, auquel participa la compagnie de Bernard Régis, permit la libération des prisonniers et la récupération du bétail razzié qui fut ensuite restitué sans rançon aux habitants de Masmolène et de Tresques. Toutefois, Jean Coq, qui agissait en tant que capitaine de la ville, fit donner un agneau à chaque Tuchin qui avait pris part à cet affrontement. Ce geste semble avoir rencontré l'adhésion des propriétaires des troupeaux qui se réjouirent et affirmèrent qu'ils aimaient mieux que les habitants de Bagnols mangent tous leurs animaux plutôt qu'ils ne restent au pouvoir des troupes de Gantonnet d'Apzac $^{67}$. De même, les habitants de Tresques firent donner une ou deux salmées de farine aux Tuchins et ceux de Masmolène leur remirent une somme d'argent pour les remercier de leur intervention ${ }^{68}$. Certes, les sources ne nous permettent guère de savoir si ces dons ne dissimulent pas une forme de rançon versée en nature et qui serait la contrepartie du travail opéré par les Tuchins. Cependant, les villageois de Tresques et de Masmolène ne pouvaient qu'y consentir dans la mesure où cette participation 
représentait une charge financière bien moindre que celle qu'ils auraient dû verser aux routiers pour récupérer leurs troupeaux et leurs pâtres.

Les confiscations opérées par les Tuchins sur les troupeaux d'ovins récupérés au détriment des gens d'armes s'apparentent, tout compte fait, à un détournement du prélèvement seigneurial au profit de ceux qui, devant la carence des pouvoirs traditionnels et leur incapacité à assurer la protection des individus et de leurs biens, ont pris eux-mêmes en charge la défense des communautés. En tout état de cause, les propriétaires du bétail furent placés devant le fait accompli. Ce n'est en effet que trois ou quatre jours après le combat qu'ils parvinrent dans la ville pour récupérer leur bien et, durant ce laps de temps, les hommes de Bernard Régis qui se trouvaient au couvent des Frères Mineurs de Bagnols-sur-Cèze en profitèrent pour consommer les agneaux qui leur avaient été offerts par Jean Coq $^{69}$. La révolte débouche une fois de plus sur le renforcement des liens de sociabilité, à travers la célébration d'un festin au cours duquel Bagnolais et Tuchins mangèrent côte à côte dans une sorte de méchoui improvisé, de la même manière qu'ils avaient combattu ensemble ${ }^{70}$. Le partage de la nourriture, comme celui du vin, vient concrétiser cette nécessaire solidarité qu'exprimait déjà le compagnonnage militaire, lequel avait rendu possible la restitution des troupeaux à leurs légitimes propriétaires. Le repas pris en commun s'affirme également comme une forme de redistribution égalitaire qui a pour objectif de renforcer le lien social aussi bien à l'intérieur même du milieu des révoltés qu'en direction des "populaires» de la ville gravitant autour du mouvement et des élites urbaines. Les Tuchins recréent donc à cette occasion une solidarité qui dépasse le cadre interne de leur organisation et redonnent au terme de compagnon son sens le plus élémentaire. La sauvegarde du bétail nécessaire à la survie des communautés, celle des biens et des personnes, prime alors sur les antagonismes sociaux et cet objectif commun manifeste l'existence des liens d'entraide qui unissent les habitants face à leurs agresseurs. La défense du territoire explique largement l'indispensable recours aux Tuchins et la popularité dont bénéficia ce mouvement au sein de populations soumises à de constantes pressions de la part des gens d'armes.

Cette succession de razzias et de contre-razzias illustre bien la guerre que menèrent Tuchins et gens d'armes dans les vigueries de Bagnols-sur-Cèze et d'Uzès entre novembre 1382 et mai 1383. Un jugé du Parlement de Paris sanctionnant un procès mû par Jean Cabassole contre les consuls de Saint-Laurent-des-Arbres ${ }^{71}$ fournit un autre exemple de ces alliances entre autorités urbaines et Tuchinat. Après l'occupation du lieu par les hommes de Ferragut, les soldats de Gantonnet d'Apzac vinrent se loger à Saint-Geniès-de-Comolas ${ }^{22}$ et le lieutenant du vicomte de Turenne interdit aux habitants de Saint-Laurent-des-Arbres de recevoir dorénavant des Tuchins à l'intérieur de leurs murs, sous peine d'être défiés à feu et à sang. Toutefois, devant la menace que faisaient peser sur leur communauté les courses des routiers, les procureurs de SaintLaurent-des-Arbres résolurent de faire appel aux Tuchins et écrivirent en ce sens aux consuls de Laudun ${ }^{73}$. La compagnie de Verchière, qui vint à leur rescousse, fut accueillie avec un certain soulagement par les habitants : les Tuchins mirent le village en défense et montèrent contre Saint-Geniès-de-Comolas une expédition qui avait pour but de récupérer une partie du bétail dérobé aux habitants. Un tel appel au secours de la part des communautés suppose un vaste réseau de complicités couvrant pour le moins les vigueries d'Uzès, de Roquemaure et de Bagnols-sur-Cèze ainsi qu'une certaine rapidité dans la transmission des informations. Les lettres échangées entre les consuls de Saint- 
Laurent-des-Arbres et ceux de Laudun indiquent la large part de responsabilité que portent les oligarchies urbaines dans l'implantation et le développement du Tuchinat.

Le refus de tout compromis et la politique de terreur menée par les troupes de Gantonnet d'Apzac et du vicomte de Turenne sont donc largement responsables de l'aggravation de la situation. Alors que, dans un premier temps, consulats et syndicats semblent s'être montrés très réservés vis-à-vis des Tuchins dont la connotation antifiscale et la dimension sociale paraissaient trop prononcées à leurs yeux, le comportement des gens d'armes censés représenter l'autorité du duc de Berry ne laissait d'autre choix aux communautés que de s'allier avec les Tuchins pour préserver un certain équilibre tant économique que social. Les Tuchins représentaient alors dans cette partie du Languedoc la seule force militaire capable de tenir tête aux compagnies et d'opérer des raids aboutissant à la récupération d'une partie du bétail et à la libération des prisonniers. En même temps, ils étaient sans doute chargés d'assurer une certaine protection aux travailleurs agricoles et permettaient d'éviter une interruption totale des travaux des champs, laquelle se serait révélée catastrophique en termes de ravitaillement. C'est bien la survie physique des villages languedociens qui est ici en jeu et rend inévitable le recours aux Tuchins, dont l'objectif primordial reste bien la lutte contre les routiers, fussent-ils au service du lieutenant du roi en Languedoc. À plusieurs reprises en effet, Verchière, Ferragut, Vachon et Bernard Régis réaffirment le désir de leurs compagnons de mettre un terme à leur mouvement, de rentrer chez eux et de vivre en paix, à condition toutefois que les gens d'armes cessent d'incendier leurs villages, de s'emparer de leurs troupeaux et de leurs récoltes et de capturer certains d'entre eux pour les soumettre à rançon.

La révolte des Tuchins n'est d'ailleurs pas qu'un simple compagnonnage d'armes, puisque ses membres ne participent que par intermittence aux opérations menées sous la direction de leurs capitaines. Le Tuchinat est peut-être une véritable fraternité de loisir et de travail avant même d'être un mouvement politique et social. Entre deux actions militaires, les compagnons retournent à leur état premier de brassiers et leur solidarité se forge aussi à l'aune d'un labeur en commun. Tout au long de l'insurrection, les moments forts du travail agricole viennent scander l'activité des Tuchins. Outre les moissons qui voient les révoltés troquer leur épée pour une faucille, ce sont essentiellement, dans une région à forte prédominance viticole, les travaux de la vigne qui mobilisent les compagnons d'un jour rendus momentanément à la vie civile. Putatio, fossura, binatio, vindemia ${ }^{74}$, les quatre opérations fondamentales de la culture de la vigne mobilisent ainsi au service des propriétaires terriens que peuvent être les boni homines de Bagnols-sur-Cèze une main-d'œuvre pour laquelle travail agricole et revendication sociale ne sont que les deux faces d'un même phénomène d'intégration au sein des sociabilités villageoises. L'ayssada, cette houe que les vignerons languedociens utilisent pour fouir la terre et déchausser les pieds de vigne, est, au même titre que l'épée des Tuchins, un symbole de reconnaissance sociale que partagent entre eux les compagnons. C'est d'ailleurs cette houe que porte sur l'épaule le laboureur Jacques Fabre se rendant à la vigne de son père qui incite Verchière à ses hommes à en faire l'une de leurs nouvelles recrues ${ }^{75}$.

21 Cette notion de convivialité et de familiarité qui paraît bien être au cœur du mouvement des Tuchins ne s'exprime pas seulement au travers d'un travail accompli en commun mais également par le biais de rites de sociabilité qui investissent l'espace urbain. De nombreux boni homines, à l'instar du drapier Laurent Raynouard, n'ont 
retenu de la présence des compagnons à Bagnols-sur-Cèze que la fréquentation du lupanar de la ville et le fait que certains des révoltés n'hésitaient pas à se promener dans les rues au bras de prostituées, ce qui à leurs yeux constitue une grave transgression des règles qui assurent la cohésion de la communauté. D'autres bourgeois de la cité préfèrent, quant à eux, insister sur les longs séjours que faisaient les révoltés au sein des tavernes et sur leur propension à jouer aux dés l'argent issu de leurs pillages. Ils tentent ainsi, au travers des réponses fournies aux questions des commissaires royaux, de définir les Tuchins par des traits de caractère qui les assimilent au groupe de ces juvenes qui appuient leur identité sur ces lieux essentiels de sociabilité que constituent l'auberge et le bordel municipal. Alacriter vivere ${ }^{76}$, telle est d'ailleurs l'expression par laquelle deux Tuchins présents à Aiguèze définissent leur mode de vie depuis leur entrée dans le mouvement. Cette volonté affirmée par les compagnons d'occuper le devant de la scène et d'étaler aux yeux de tous une sociabilité d'ordinaire reléguée au second plan se traduit notamment par la prise de possession des tavernes et du lupanar : causa recreationis, c'est ainsi que le capitaine Bernard Régis définit l'action de ses hommes dans la ville. Toutefois, cette réoccupation de la ville n'est pas que délassement, elle est aussi une réappropriation symbolique de l'espace urbain et de ses lieux de sociabilité. Cette solidarité paysanne s'exprime à travers la fréquentation du bordel et des tavernes qui deviennent des espaces de rencontre privilégiés, où les Tuchins venus d'horizons différents et de villages éloignés, se retrouvent non seulement pour redonner un sens à leur action mais aussi pour définir leur stratégie et coordonner de nouvelles opérations. Selon plusieurs témoins, c'est à Bagnols-sur-Cèze dans les tavernes de la Cloche Blanche et de la Roussette que les Tuchins auraient mis au point leur plan pour s'emparer du château d'Aiguèze. Manger, boire et jouer ensemble - aux dés par exemple - sont des signes élémentaires de la sociabilité villageoise qui viennent ici s'enraciner dans l'espace urbain. La révolte est bien une fête, "une rupture de la vie quotidienne »", et en ce sens elle est dissipation rapide du butin accumulé. Les abondantes fournitures de victuailles et de vin dont bénéficient les Tuchins dans les premiers temps de leur installation à Pont-Saint-Esprit et à Bagnols-sur-Cèze, marquées en particulier par la présence de viande - plats de tripes, agneaux prélevés sur les troupeaux razziés ou moutons - sont immédiatement consommées parce que les repas pris en commun permettent de refonder le lien social et le compagnonnage militaire qui est à la base du Tuchinat. Tous les témoins issus de l'oligarchie urbaine ont été frappés de cette prodigalité, d'autant plus extraordinaire qu'elle paraitt trancher avec l'habituel sens de l'économie des communautés paysannes. Consumare et discipare, telle est la formule par laquelle les boni homines résument le comportement des Tuchins au sein de l'espace urbain. À l'occasion de leurs témoignages, les boni homines opèrent néanmoins un détournement qui consiste à nier les rites de sociabilité intrinsèques au Tuchinat et à ne voir que gaspillage dans ce qui est refondation du lien social.

22 À travers l'exemple de Bagnols-sur-Cèze et des villages environnants, l'insurrection des Tuchins révèle ainsi la force du lien social qui permet d'assurer la cohésion et la survie des villages languedociens. C'est par les communautés et à travers elles que la révolte prend forme et le mouvement épouse les structures de sociabilité et les liens de voisinage qui donnent tout leur sens à l'entité villageoise. Le Tuchinat ne doit d'ailleurs son implantation durable et son succès qu'à sa capacité à s'insérer à l'intérieur des cadres communautaires et à les faire fonctionner à son profit. Toutefois, il ne trouve à s'exprimer qu'en raison de la défaillance même de ces cadres et de leur incapacité à 
assurer la défense des populations concernées. Dans le Comtat Venaissin pourtant tout proche et soumis aux mêmes pressions de la part des gens d'armes, c'est " sous l'effet des injonctions des autorités et de la mobilisation des habitants ${ }^{78}$ que le pays put être mis en défense. A contrario, dans le Languedoc de cette fin du XIV ${ }^{e}$ siècle, ce sont les villageois eux-mêmes qui prennent en charge la lutte contre les compagnies, dût-elle se faire à l'encontre des autorités seigneuriales, urbaines ou royales. Ceci ne signifie pourtant en aucun cas que le Tuchinat puisse être considéré comme un défi lancé directement au roi puisque jamais les compagnons ne remettent en cause la légitimité de ce dernier à gouverner la province. En ce sens, bien plus qu'une révolte, le Tuchinat est d'abord et avant tout un mouvement d'autodéfense de villageois exaspérés par la charge humaine et financière que représente la guerre pour ceux qui la subissent et, s'inscrit dans cette longue succession de "guerres du peuple " qui scandent les XIV ${ }^{\mathrm{e}}$ et $\mathrm{XV}^{\mathrm{e}}$ siècles.

\section{NOTES}

1.M. Mollat et Ph. Wolff, Les Révolutions populaires en Europe aux XIV et XV siècles, Paris, Calmann-Lévy, 1970, p. 185.

2.Chronique du Religieux de Saint-Denis concernant le règne de Charles VI de 1380 à 1422, éd. Bellaguet, Paris, 1839-1852, t. I, p. 310. Sur l'utilisation du terme d'abjectissimi dans l'œuvre du Religieux de Saint-Denis, voir B. Guenée, L'opinion publique à la fin du Moyen Âge d'après la "Chronique de Charles VI » du Religieux de Saint-Denis, Paris, Perrin, 2002, p. 107. Bernard Guenée note que ce vocable est rarement employé et sert toujours à désigner des «moments de violence où des hommes de bas état sont sortis de l'ombre et ont fait peur ".

3.C. Gauvard, « Les révoltes du règne de Charles VI : tentative pour expliquer un échec ", Révolte et Société, Paris, 1989, p. 54.

4.B. Geremek, Les Marginaux parisiens aux XIVe et XVe siècles, Paris, Flammarion, 1976, p. 7.

5.Je dois ces remarques, formulées lors de ma soutenance de thèse, à M. Albert Rigaudière que je remercie ici vivement.

6.C. Gauvard, « Le concept de marginalité au Moyen Âge : criminels et marginaux en France aux XIV et $\mathrm{XV}^{\mathrm{e}}$ siècles », Histoire et criminalité de l'Antiquité au XXe siècle : nouvelles approches, Dijon, 1992, pp. 362-368.

7.Walter Von Warburg, Französisches Etymologisches Wörterbuch, Basel, 1966, t. 13. Au mot tuscus.

8.R. Fossier, Sources de l'histoire économique et sociale du Moyen Âge occidental, Tournai, Brepols, 1999, p. 23.

9.AN, JJ 108, n 259, fol. 146 ; cité par S. Luce, Histoire de Bertrand Du Guesclin et de son époque, Paris, 1876, p. 273.

10.G. Lefèvre-Pontalis, «Épisodes de l'invasion anglaise : la guerre de partisans dans la Haute-Normandie (1424-1429) », Bibliothèque de l'École des Chartes, t. LIV, 1893, pp. 475-521. 
11.AN, JJ 98, n 138, fol. 42 ; Vieille-Brioude, c. et arr. Brioude, Haute-Loire. 12.P. Charbonnier, "Qui furent les Tuchins? ", Violence et Contestation au Moyen Âge, Actes du $114^{\mathrm{e}}$ Congrès National des Sociétés Savantes (Paris, 1989), Paris, 1990, pp. 235-247.

13.Chronique du Religieux de Saint-Denis..., t. I, p. 306 : Qui...mutuo se adunantes, terribilibus sacramentis sese astrinxerant, quod deinceps nullo jugo subsidiorum colla submiterent. 14.Pour le corpus des lettres de rémission délivrées par la chancellerie royale à l'occasion du Tuchinat tant auvergnat que languedocien, je me permets de renvoyer à ma propre thèse : $\mathrm{V}$. Challet, Mundare et aufere malas erbas : la révolte des Tuchins en Languedoc (1381-1384), Université de Paris-I Panthéon-Sorbonne, 2002, vol. I, pp. 29-38. 15.Wace, Roman de Rou, éd. A. J. Holden, Paris, Société des Anciens Textes Français, 1970-1973 : « Allions nous par un serment/Nous et nos biens nous défendons/et tous ensemble nous tenons "; exemple cité dans M. Bourin et R. Durand, Vivre au village au Moyen Âge : les solidarités paysannes $d u X^{e}$ au XIII siècle, Rennes, Presses Universitaires de Rennes, 1984, p. 191.

16.Ad jurandum ut esset eis bonus et fidelis. Il s'agit d'un témoignage recueilli dans le cadre du procès intenté en 1385 par Pons Biordon, ancien syndic de Pont-Saint-Esprit, ancien visteur général de la gabelle du sel et seigneur d'Aiguèze, contre la communauté de Bagnols-sur-Cèze. Les actes de ce procès sont conservés aux Archives Municipales de Bagnols-sur-Cèze (FF 11 et FF 11 bis) et ont été intégralement publiés dans le cadre de ma thèse.

17.Bene et fideliter se habere; V. Challet, op. cit., Annexes, volume II, p. 220.

18.G. Lefevre-Pontalis, op. cit. : ces partisans se regroupent en compagnies et prêtent un serment : «Auquel fist faire serement de le servir bien et loyaulment » (AN JJ 173, $\left.\mathrm{n}^{\circ} 534\right)$.

19.AN, JJ 137, n 107 ; publié par A. Thomas, « Dans les jardins d'Arpaillargues, en 1397 : dernier écho de la Touchinerie du Bas-Languedoc ", Annales du Midi, 1914, pp. 232-241. 20.Bagnols-sur-Cèze, com. et c., arr. Nîmes, Gard.

21.Ce phénomène se retrouve pour l'ensemble des révoltes de l'époque médiévale. Pour n'en citer qu'un seul exemple, le terme de Ciompi, sobriquet qui fut appliqué aux ouvriers florentins en 1378, renverrait à l'idée de bâtardise. Voir R. Paris, « Les Ciompi : cardeurs, foulons, bâtards ? ", Médiévales, n³0, printemps 1996, pp. 109-114.

22.On y trouve indifféremment l'expression Tochis et companhos Tochis; M. Boudet, Registres consulaires de Saint-Flour (1376-1405), Paris, Champion, 1898, p. 99, 104, 185. Sur les relations entre Tuchins et pouvoir consulaire à Saint-Flour, voir A. Rigaudière, SaintFlour, ville d'Auvergne au Bas Moyen-Âge : étude d'histoire administrative et financière, Paris, P.U.F., 1982, t. II, pp. 638-640.

23.AD de l'Aveyron, 2 E 178-8, fol. $4^{\mathrm{r}}, 7^{\mathrm{r}}, 9^{\mathrm{r}}, 11^{\mathrm{v}}$. Najac, com. et c., arr. Villefranche-deRouergue, Aveyron.

24.AD de l'Aveyron, 2 E 67-22, comptes non foliotés. Conques, com. et c., arr. Rodez, Aveyron.

25.Saint-Gervais, c. Bagnols-sur-Cèze, arr. Nîmes, Gard.

26.V. Challet, op. cit., Annexes, vol. I, p. 202.

27.N. Wright, Knights and peasants: the Hundred Years War in the French countryside, Boydell Press, Woodbridge, 1998, p. 5.

28.Dès 1369, des chefs de compagnie notoires comme Noli Papillon, Antoine bâtard de Terride ou Benoît Chapparel furent retenus au service du duc d'Anjou, lieutenant du roi 
en Languedoc. Voir Ph. Contamine, Guerre, État et Société à la fin du Moyen-Âge : étude sur les armées des rois de France (1337-1494), Paris-La Haye, Mouton, 1972, p. 166.

29.Honoré Bovet, L'Arbre des batailles, BNF, ms. fr. 487, fol. $74^{\mathrm{r}}$.

30.N. Wright, op. cit., pp. 80-93.

31.N. Gonthier, Le Châtiment du crime au Moyen Âge, Rennes, P.U.R., 1998, p. 85.

32.AN, JJ 93, n 181 ; publiée dans C. de Vic et J. Vaissète, Histoire générale du Languedoc, Toulouse, 1889, t. X, preuves, col. 1304-1306. Chusclan, c. Bagnols-sur-Cèze, arr. Nîmes, Gard.

33.Les comptes de Barthélémy des Noces, trésorier des guerres de Jean de Berry en tant que lieutenant du roi, permettent d'établir qu'au moment de pénétrer en Languedoc, le duc de Berry disposait d'un contingent de 560 arbalétriers et de 1240 hommes d'armes. BnF, ms. fr. 32510 , fol. $247^{\mathrm{r}}-248^{\mathrm{r}}$. Sur l'action de Jean de Berry en Languedoc, voir F. Lehoux, Jean de France, duc de Berri. Sa vie. Son action politique (1340-1416), Paris, Picard, 1966, t. II, pp. 9-108.

34.Vénéjan, c. Bagnols-sur-Cèze, arr. de Nîmes, Gard.

35.Saint-Michel-d'Euzet, c. Bagnols-sur-Cèze, arr. Nîmes, Gard.

36.Ferragut est, sans doute, de tous les capitaines tuchins, celui qui connut le destin le plus exceptionnel. Il devait par la suite commander les troupes qui entrèrent en Arles en 1384. Voir L. Stouff, « Une ville de France entre Charles de Duras et les Angevins. L'entrée des Tuchins dans Arles le 24 juillet 1384 », 1388 : La dédition de Nice à la Savoie, Paris, Publications de la Sorbonne, 1990, pp. 144-157.

37.Une salmée équivaut à quatre setiers, le setier valant lui-même douze boisseaux.

38.V. Challet, op. cit., Annexes, vol. I, p. 378. Jean Portal est originaire de Saint-Montant, c. Bourg-Saint-Andéol, arr. Privat, Ardèche.

39.Vexillis erectis et trompetis sonantibus. Témoignage de Pons de Bonvent, drapier de Bagnols-sur-Cèze, cité dans V. Challet, op. cit., Annexes, vol. I, p. 174.

40.Il s'agit de la rue que les documents qualifient de carreria recta. Cette rue était la plus importante et la plus riche des artères de Bagnols-sur-Cèze d'après P. Béraud, Histoire de la ville de Bagnols-sur-Cèze, Nîmes, Éd. de la Maison Carrée, 1941, p. 47.

41.A. Stella, La Révolte des Ciompi, Éditions de l'EHESS, Paris, 1993, p. 69.

42.Sur l'importance des bruits dans les rituels, voir N. Offenstadt, «Cris et cloches. L'expression sonore dans les rituels de paix à la fin du Moyen Âge », Hypothèses 1997, Paris, Publications de la Sorbonne, 1998, pp. 51-58.

43.Sur ces cérémonies voir $\mathrm{B}$. Guenée et $\mathrm{F}$. Lehoux, Les entrées royales françaises de 1328 à 1515, Paris, Éditions du CNRS, 1968 et pour un espace géographique plus proche

N. Coulet, « Les entrées solennelles en Provence au XIV siècle : aspects nouveaux sur les entrées royales françaises au bas Moyen Âge ", Ethnologie Française, t. VII (1), 1977, pp. 63-82.

44.Sur le fonctionnement des institutions urbaines à Bagnols-sur-Cèze, voir P. Béraud, op. cit, pp. 36-41.

45.L'hôtel que le vicomte de Turenne avait fait acheter le $1^{\text {er }}$ mai 1378 et qui constituait sa résidence lorsqu'il venait à Bagnols-sur-Cèze se trouvait dans la rue Bone Vite, autrement appelée Ad Furnum Regis. L'acte de vente se trouve dans le registre de Guillaume de Collorgue, notaire de la ville (AD du Gard, 2 E 14-18, fol. 101 ${ }^{\mathrm{v}}$ )

46.Sed dictus dominus vicecomes ante quam a dicta villa discederet fuerat loqutus cum dicto Vercheria et quibusdam aliis Tuchinis, dicendo eisdem quod agerent bonum et presertim dicto Vercherie qui fuerat officiarius patris ipsius domini vicecomitis. Témoignage de Pons de Bonvent, cité dans V. Challet, op. cit., Annexes, vol. I, p. 174. 
47.Il s'agit de Jacme Blausac qui avait dit que « les Touchins estoient bon compaignons et ce qu'ils faisoient estoit bien fait ». BnF, ms. lat. 9176 ; publié dans C. De Vic et J. Vaissète, op. cit., t. X, col. 1673.

48.BnF, Clairambault 957, $\mathrm{n}^{\circ} 45$; cité par F. Lehoux, op. cit., t. II, p. 78.

49.J. Favier, La Guerre de Cent Ans, Paris, Fayard, 1980, p. 447.

50.Journal d'un bourgeois de Paris (1405-1449), A. Tuetey éd., Paris, 1881, p. 110.

51.C. Gauvard, « De grace especial ». Crime, État et société en France à la fin du Moyen Âge, Paris, Publications de la Sorbonne, 1991, t. II, p. 570.

52.Et quod ipse dominus vicecomes miserat quesitum Ferragutum, sed ipse Ferragutus noluerat venire loqutum cum eodem. Témoignage de Jean Coq, marchand de Bagnols-sur-Cèze et capitaine de la ville au moment des faits, cité dans V. Challet, op. cit., Annexes, vol. I, p. 188.

53. Qui frater Johannes de Ferranis ipsum loquentem salutavit et invitavit pro potu, nam ipsius loquentis noticiam habebat. Témoignage de frère Guillaume Vital, cité dans V. Challet, op. cit., Annexes, vol. I, p. 131.

54.Saint-Paulet-de-Caisson, c. Pont-Saint-Esprit, arr. Nîmes, Gard.

55.Dictis Tuchinis idem Petrus Gaydelli de eius vino albo dedit ad bibendum; et cum dictus Vercheria gustasset, noluit multum bibere de eodem, dicens quod non erat bonum. Témoignage de Pons Antoine, prêtre d'Aiguèze, cité dans V. Challet, op. cit., Annexes, vol. I, p. 379. 56.C. Gauvard, "Cuisine et paix à la fin du Moyen-Âge ", La Sociabilité à table : commensalité et convivialité à travers les âges, Rouen, 1990, pp. 325-334.

57.Saint-Gervais, canton de Bagnols-sur-Cèze, arrondissement de Nîmes, Gard.

58. Cité dans V. Challet, op. cit., Annexes, vol. I, p. 181, 216 et 229.

59.Ibidem, p. 270.

60.Ibidem, p. 237.

61.Ibidem, p. 200.

62.Ibidem, p. 33.

63.Les comptes consulaires de la ville contiennent plusieurs mentions de paiements effectués « als homes d'armas que s'apelavan Tochis » en novembre et décembre 1381. Le registre des comptes pour l'année 1381-1382 (AM de Narbonne, CC 2599) est manquant mais des extraits en ont été publiés par A. Blanc, Le Livre de comptes de Jacme Olivier, marchand narbonnais du XIV ${ }^{e}$ siècle, Paris, Picard, 1899, t. II, pp. 949-952.

64.Sur les débuts de la carrière de ce personnage, voir H. Kaminsky, « The early career of Simon de Cramaud ", Speculum, vol. 49 (3), 1974, pp. 499-534.

65.Masmolène, c. Uzès, arr. Nîmes, Gard ; Tresques, c. Bagnols-sur-Cèze, arr. Nîmes, Gard.

66. Cité dans V. Challet, op. cit., Annexes, Vol. I, p. 127 et 162. Plusieurs des habitants de Bagnols qui s'étaient auparavant joints aux Tuchins et avaient pour lors quitté le mouvement se joignirent à cette opération.

67.p. 162.

68.Ibidem, p. 103 et 162 .

69.Ibidem, p. 154 et 238.

70.Sur le rôle du repas pris en commun comme élément fondateur de la paix sociale, voir C. Gauvard, « Cuisine et paix à la fin du Moyen Âge », op. cit., pp. 325-334.

71.AN, X 1 A 39, n 3, fol. $268^{\text {vo; }}$ Saint-Laurent-des-Arbres, c. Roquemaure, arr. Nîmes, Gard.

72.Saint-Geniès-de-Comolas, c. Roquemaure, arr. Nîmes, Gard.

73.Laudun, c. Bagnols-sur-Cèze, arr. Nîmes, Gard. 
74.La putatio correspond à la taille des plants qui se fait à la serpe en janvier ou en février ; la fossura est le déchaussage des pieds qui se pratique en février ou en mars ; la binatio ou binage est réalisée en mai ou en juin. Les vendanges, elles, se déroulent lors du mois de septembre.

75.Témoignage de Jacques Fabre, laboureur de Bagnols-sur-Cèze ; cité dans V. Challet, op. cit., Annexes, vol. I, p. 152.

76.Témoignage de Bertrand Fournier, sergent royal de Bagnols, qui rapporte des paroles prononcées par deux Tuchins présents à Aiguèze ; cité dans V. Challet, op. cit., Annexes, vol. I, p. 73.

77.Y.-M. Bercé, Histoire des Croquants, Paris, Seuil, 1986, p. 306.

78.G. Butaud, «Villages et villageois du Comtat Venaissin en temps de guerre (milieu $\mathrm{XIV}^{\mathrm{e}}$ - début XV $\mathrm{XV}^{\mathrm{e}}$ siècle) », Les Villageois face à la guerre (XIVe-XVIII siècles), Cahiers de Flaran XXII, Toulouse, Privat, 2002, p. 63.

79.J.-L. Roch, « Les Guerres du peuple : autodéfense, révolte et pillage dans la Guerre de Cent Ans ", Images de la Guerre, J. Maurice, D. Couty et M. Guéret-Laferté éd., Paris, Presses Universitaires de France, 2002, pp. 47-61.

\section{AUTEUR}

\section{VINCENT CHALLET}

Université Paul-Valéry Montpellier-III 